\title{
Evaluación formativa: una revisión sistemática de estudios en aula
}

\author{
Formative assessment: a systematic review studies in the classroom \\ Avaliação formativa: uma revisão sistemática dos estudos em sala de aula
}

Wilfredo Hernán Bizarro Flores

wbizarrof@unmsm.edu.pe

https://orcid.org/0000-0003-0267-6322

\author{
Pilar Julia Paucar Miranda \\ ppaucarm@unmsm.edu.pe \\ https://orcid.org/0000-0001-9141-4986
}

\author{
Edith Chambi-Mescco \\ echambim@unmsm.edu.pe \\ https://orcid.org/0000-0002-0535-5906
}

Universidad Nacional Mayor de San Marcos, Lima-Perú

Recibido 23 de abril 2021 | Arbitrado y aceptado 25 de mayo 2021 | Publicado en 01 julio 2021

\section{RESUMEN}

El objetivo del presente artículo fue analizar la evaluación formativa a través de una revisión sistemática de estudios desarrollados en aula y producidos en los últimos cinco años; se tomó la base de datos de Scopus, Sciencedirect y Scielo. El estudio documental radicó en la selección de artículos científicos referentes a la aplicación de la evaluación en aula por docentes e investigadores, desde el nivel inicial de la educación básica hasta la superior universitaria. Se encontró que la evaluación formativa es una estrategia muy valiosa para desarrollar competencias; integra el proceso pedagógico a través de la comunicación permanente docente-estudiante; además incluye la retroalimentación que es esencial al momento de evaluar. Se concluye que la evaluación formativa fomenta del desarrollo integral del estudiante, causa confianza, genera la autonomía, promueve la autoevaluación y la coevaluación, es participativa $y$, sobre todo, produce la mejora de los niveles de aprendizaje en los estudiantes.

Palabras clave: evaluación formativa; aula; aprendizaje; retroalimentación; revisión sistemática

\section{ABSTRACT}

The objective of this article was to analyze the formative evaluation through a systematic review of studies developed in the classroom, produced in the last five years, the Scopus, Sciencedirect and Scielo databases were taken. The documentary study was based on the selection of scientific articles referring to the application of classroom evaluation by teachers and researchers, from the initial level of basic education to university higher education. It was found that formative evaluation is one of the very valuable strategies when developing competencies, integrates the pedagogical process through permanent teacher-student communication, and feedback is essential when evaluating. It is concluded that it promotes the integral development of the student; it causes trust, generates autonomy, promotes self-evaluation and co-evaluation, is participatory and, above all, produces an improvement in student learning levels.

Key words: Formative Evaluation; Classroom; Learning; Feedback; Systematic Review

\section{RESUMO}

O objetivo deste artigo era analisar a avaliação formativa através de uma revisão sistemática dos estudos desenvolvidos em sala de aula e produzidos nos últimos cinco anos; foram utilizados os bancos de dados Scopus, Sciencedirect e Scielo. $\mathrm{O}$ estudo documental foi baseado na seleção de artigos científicos sobre a aplicação da avaliação em sala de aula por professores e pesquisadores, desde o nível inicial do ensino básico até o ensino superior. Constatou-se que a avaliação formativa é uma estratégia muito valiosa para desenvolver competências; ela integra o processo pedagógico através da comunicação permanente professor-aluno; ela também inclui feedback, que é essencial na avaliação. Conclui-se que a avaliação formativa promove o desenvolvimento integral do estudante, gera confiança, gera autonomia, promove a auto-avaliação e a co-avaliação, é participativa e, acima de tudo, produz uma melhoria nos níveis de aprendizagem dos estudantes.

Palavras-chave: Avaliação formativa; sala de aula; aprendizagem; feedback; avaliação formativa; revisão sistemática 


\section{INTRODUCCIÓN}

La evaluación, desde la Revolución Industrial, estaba pensada en seleccionar personas para el trabajo como si fuéramos todos iguales; esta concepción ha venido formando parte de la práctica docente. Sin embargo, evaluar, en estos tiempos de la sociedad del conocimiento, se enfoca en la diversidad, asumiendo que todos somos diferentes, que se tiene que distintas formas de aprender, estilos y ritmos de aprendizaje; por tanto, también debe ser evaluados de diferentes formas. La evaluación formativa tiene como fin mejorar y motivar la autonomía en el estudiante, pues ayuda a que se dé cuenta por sí mismo de sus logros y dificultades, que reflexione sobre sus errores. Además, le ayuda al docente a progresar a través de estrategias y prácticas evaluativas de preguntas, repreguntas, tareas auténticas, retroalimentación de esas tareas y ver cómo va mejorando su quehacer educativo en el tiempo.

Se pensaba que la evaluación formativa tenía que ver con hacer una devolución "correcta" de las notas obtenidas, es decir, si las calificaciones estaban bien especificadas y los errores bien detallados, ya era suficiente (Ravela y Cardoner, 2019). Esto ocurre fundamentalmente porque es lo se ha vivido durante más de 15 años como estudiantes de primaria, secundaria y universidad, y lo que se ha seguido viviendo a lo largo de toda la carrera profesional como docentes (López-Pastor y SuyapaMartínez, 2019). Hoy "uno de los principales retos al que se enfrenta el profesor es cambiar de modelos tradicionales de evaluación, claramente centrados en los exámenes y la calificación, a modelos de evaluación formativa, claramente centrados en la mejora de los aprendizajes" (López-Pastor, 2017).
Ello implica la transformación de las prácticas tradicionales de evaluación en aula (Bizarro, Sucari y Quispe-Coaquira, 2019), pues los resultados de la investigación educativa, en los últimos 40 años, han revelado que los métodos de enseñanza tradicionales basados en la exposición de conocimientos son poco efectivos en el desarrollo de aprendizajes significativos (Talanquer, 2015). Consecuentemente la práctica evaluativa está peor aún relegada.

La labor del docente para implementar la evaluación formativa en aula no es nada fácil, puesto que para poner en práctica este proceso se necesita experiencia, pero sucede que el docente nunca la ha experimentado en su formación inicial y profesional (López et al., 2020). Entonces implica cambiar las prácticas evaluativas desde los conceptos hasta el manejo de instrumentos (Bizarro, Sucari, y QuispeCoaquira, 2019). Asimismo, es necesario cambiar la forma de enseñar.

El estudiante le proporciona al profesor la evidencia de cuáles son sus necesidades, lo cual le permite a este último realizar los ajustes necesarios en su modo de enseñar (Popham, 2013), puesto que en aula el papel de la retroalimentación del docente es primordial (Hattie, 2009; Hattie y Timperley, 2007; Shute, 2008; Tunstall y Gipps, 1996; Wiggins, 1989). Además, la evaluación en aula entre pares y autoevaluación mejoran la autorregulación, motivación y autoeficacia entre alumnos (Proust y Stiggins, 2002).

González y Fortoul (2016) afirman que el uso coherente de la evaluación formativa ayuda a los estudiantes a enfrentar los problemas que se presentan para desempeñarse mejor. Tiene importantes implicaciones a nivel de aula en aquellos estudiantes que tienen dificultades para 
demostrar su adquisición de competencias en los sistemas tradicionales de evaluación (Núñez-Peña, Bono, y Suárez-Pellicioni, 2015). El uso de diferentes formas de evaluación alienta a los estudiantes a ser más responsables y reflexivos (Dochy, Segers, y Sluijsmans, 2012). De modo tal hay un cambio de actitud en su evaluación (Black y Wiliam, 1998; Breckler y Wiggins, 1989). Docentes y estudiantes tienen una ardua tarea de cambiar las formas de evaluar tradicionales a la evaluación formativa. Evaluar para aprender es la misión principal. Si evaluar es una actividad natural en el ser humano (Ravela, 2017); si, en el campo de la educación de trabajo en aula, es evaluar para aprender (LópezPastor, 2017); entonces el enfoque de la evaluación formativa es contribuir al desarrollo de las competencias en los estudiantes en aula, pues es ayudar a que logren desempeños, así como corregir los errores a tiempo para mejorar los aprendizajes.

Para saber qué investigaciones existen sobre el tema, se planteó el objetivo: describir la evaluación formativa para identificar su impacto en el aprendizaje de los estudiantes, desde el análisis de los artículos producidos en los últimos cinco años, a través de la revisión sistemática en las bases de datos de Scopus, Sciencedirect y Scielo.

\section{MÉTODO}

La metodología utilizada fue la revisión sistemática con un nivel descriptivo, se realizó una síntesis de las investigaciones seleccionados de los artículos nacionales e internacionales sobre la aplicación de la evaluación formativa por docentes e investigadores en aula. Se efectuó una búsqueda sistemática de artículos publicados desde enero de 2016 hasta julio de 2020 en inglés, español o portugués, en las bases de datos: Scopus, Sciencedirect y Scielo. Se utilizaron las palabras clave: "evaluación formativa", "Formative assessment", "Avaliação formativa”, “Evaluación formativa en aula", "Formative assessment in the classroom", "Avaliação formativa em sala de aula". Con esta búsqueda se encontraron 85 artículos de las bases de datos mencionadas.

Se aplicaron criterios de inclusión de publicaciones para esta revisión: (a) estudios de evaluación formativa en aula, (b) aplicación de la evaluación en aula por docentes e investigadores, desde el nivel inicial de la educación básica hasta la superior universitaria, (c) escritos en inglés, español o portugués. Para los criterios de exclusión se tomó en cuenta la duplicidad, las temáticas diferentes y por razones justificadas, quedando seleccionados 33 artículos científicos, para ello se empleó el siguiente diagrama de flujo prisma, Figura 1: 


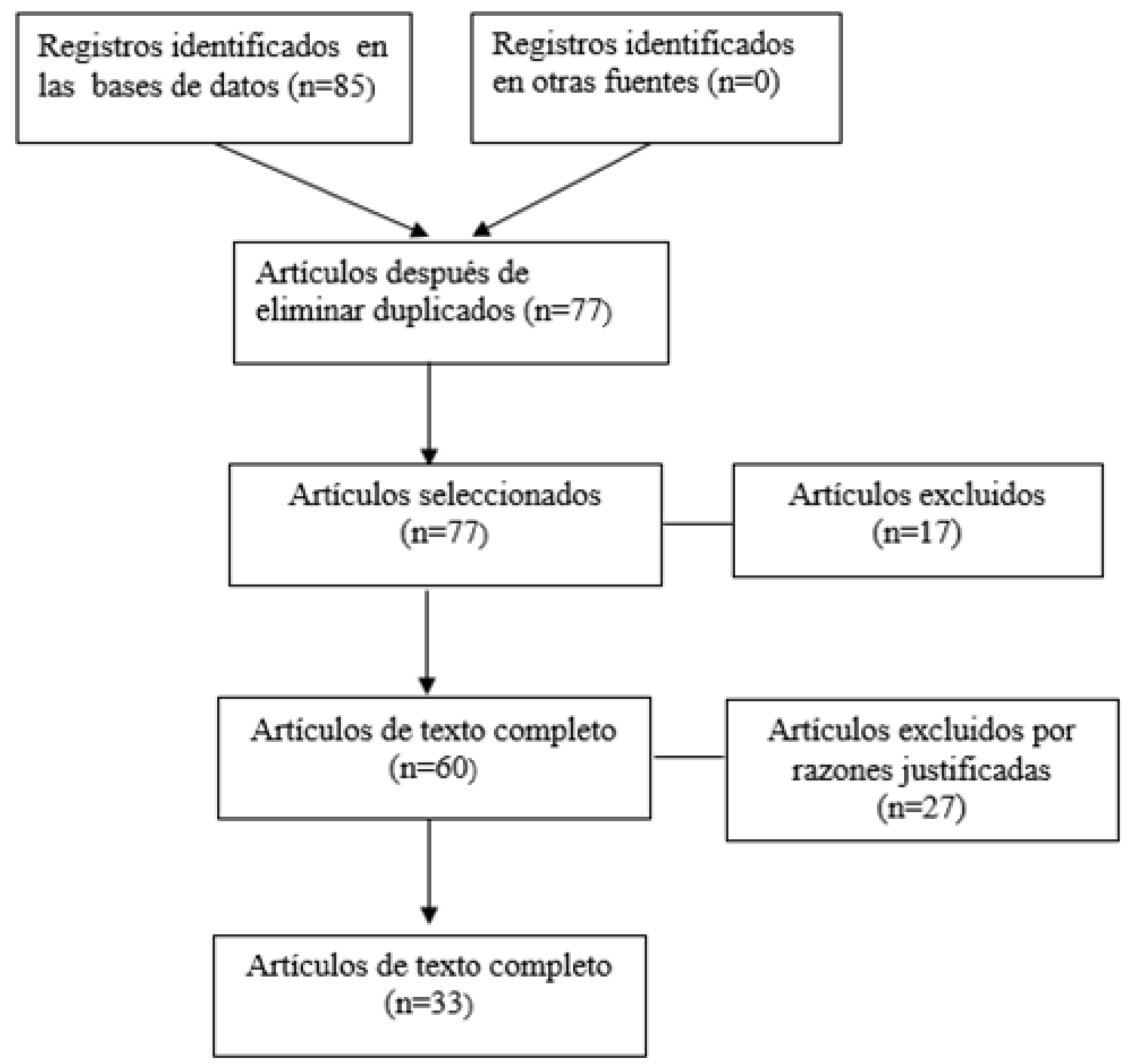

Figura 1. La búsqueda sistemática y exclusión de artículos flujo de prisma.

DESARROLLO Y DISCUSIÓN

De los 85 artículos científicos identificados, se seleccionaron 33 para este estudio, cuyos datos se observan en la Tabla 1 con categorías en uso como autores, año de publicación, la base de datos donde se encontró el artículo, revista en la que se publicó, el país, lengua y el nombre o título de la publicación. Se estudiaron 10 artículos de la base de datos Scielo, 11 artículos de Scienciedirect y 12 artículos en Scopus. 
Tabla 1. Características principales de estudios de revisión analizados.

\begin{tabular}{|c|c|c|c|c|}
\hline Autor(es). año & Base de datos & Revista & País/lengua & Nombre de la publicación \\
\hline $\begin{array}{l}\text { (Gomez, Muñoz, y } \\
\text { Londoño- } \\
\text { Vásquez, 2019) }\end{array}$ & Scielo & $\begin{array}{l}\text { Revista Aletheia, } \\
11(1), 37-68\end{array}$ & $\begin{array}{l}\text { Colombia } \\
\text { /Español }\end{array}$ & $\begin{array}{l}\text { Prácticas evaluativas en la escuela. Una } \\
\text { ruta pedagógica hacia la construcción de } \\
\text { aprendizajes significativos }\end{array}$ \\
\hline $\begin{array}{l}\text { (López Pastor et al., } \\
\text { 2020) }\end{array}$ & Scopus & $\begin{array}{l}\text { Retos, } 37 \\
\text { pp. } 620-627 \text {. }\end{array}$ & $\begin{array}{l}\text { España / } \\
\text { Español }\end{array}$ & $\begin{array}{l}\text { La importancia de utilizar la evaluación } \\
\text { formativa y compartida en la educación } \\
\text { física Formación del profesorado: proyectos } \\
\text { de aprendizaje tutorizados como ejemplo de } \\
\text { buenas prácticas }\end{array}$ \\
\hline $\begin{array}{l}\text { (Pascual-Arias, } \\
\text { García-Herranz, y } \\
\text { López-Pastor, 2019) }\end{array}$ & Scopus & $\begin{array}{l}\text { Cultura y } \\
\text { Educación }\end{array}$ & $\begin{array}{l}\text { España / } \\
\text { Español }\end{array}$ & $\begin{array}{l}\text { ¿Qué quieren los niños y niñas de Educación } \\
\text { Infantil? El papel de la evaluación formativa y } \\
\text { compartida en su derecho a decidir }\end{array}$ \\
\hline $\begin{array}{l}\text { (Rivers, Dunlosky, y } \\
\text { Joynes, 2019) }\end{array}$ & Sciencedirect & $\begin{array}{l}\text { Contemporary } \\
\text { Educational } \\
\text { Psychology }\end{array}$ & $\begin{array}{l}\text { Estados } \\
\text { Unidos. } \\
\text { Inglés }\end{array}$ & $\begin{array}{l}\text { The contribution of classroom exams } \\
\text { to formative evaluation of conceptlevel } \\
\text { knowledge }\end{array}$ \\
\hline $\begin{array}{l}\text { (Schildkamp, van } \\
\text { der Kleij, Heitink, } \\
\text { Kippers, y Veldkamp, } \\
\text { 2020) }\end{array}$ & Sciencedirect & $\begin{array}{l}\text { International Journal } \\
\text { of Educational } \\
\text { Research }\end{array}$ & $\begin{array}{l}\text { Australia. } \\
\text { Ingles }\end{array}$ & $\begin{array}{l}\text { Formative assessment: A systematic review } \\
\text { of critical teacher prerequisites for classroom } \\
\text { practice }\end{array}$ \\
\hline (Yin y Buck, 2019) & Sciencedirect & $\begin{array}{l}\text { Teaching and } \\
\text { Teacher Education } \\
\text { journal }\end{array}$ & $\begin{array}{l}\text { Estados } \\
\text { Unidos. } \\
\text { Inglés }\end{array}$ & $\begin{array}{l}\text { Using a collaborative action research approach } \\
\text { to negotiate an understanding of formative } \\
\text { assessment in an era of accountability testing }\end{array}$ \\
\hline $\begin{array}{l}\text { (Xiaotong; Chen } \\
\text { Wujin; Liao Libin; Li } \\
\text { Tian; Qin Wen y Bai, } \\
\text { 2019) }\end{array}$ & Scopus & $\begin{array}{l}\text { International Journal } \\
\text { of Morphology, } 37 \\
\text { (3), pp. 1085-1088. }\end{array}$ & $\begin{array}{l}\text { Sd } \\
\text { Inglés }\end{array}$ & $\begin{array}{l}\text { Analysis of the Teaching Effect of the Normal } \\
\text { Human Morphology with Mixed Teaching } \\
\text { Mode and Formative Evaluation in China }\end{array}$ \\
\hline (Angelini, 2016) & Scielo & $\begin{array}{l}\text { Actualidades } \\
\text { Investigativas en } \\
\text { Educación }\end{array}$ & $\begin{array}{l}\text { Costa Rica. } \\
\text { Español }\end{array}$ & $\begin{array}{l}\text { Estudio sobre la evaluación formativa y } \\
\text { compartida en la formación docente en inglés }\end{array}$ \\
\hline (Santos, 2016) & Scielo & $\begin{array}{l}\text { Río de Janeiro, v.24, } \\
\text { n. } 92, \text { p. } 637-669\end{array}$ & $\begin{array}{l}\text { Portugal. } \\
\text { Portugués }\end{array}$ & $\begin{array}{l}\text { A articulação entre a avaliação somativa } \\
\text { e a formativa, na prática pedagógica: uma } \\
\text { impossibilidade ou um desafio? }\end{array}$ \\
\hline $\begin{array}{l}\text { (Ocaña A., Pulido, y } \\
\text { Gil, 2019) }\end{array}$ & Scopus & Interdisciplinaria & Español & $\begin{array}{l}\text { Cambios en el desempeño de estudiantes de } \\
\text { pensamiento matemático desde la evaluación } \\
\text { formativa con un banco de preguntas en línea }\end{array}$ \\
\hline $\begin{array}{l}\text { (López-Benavente, } \\
\text { Ureña-Ortín, y } \\
\text { Alarcón López, } \\
\text { 2019) }\end{array}$ & Scopus & $\begin{array}{l}\text { Journal of Sport and } \\
\text { Health Research }\end{array}$ & $\begin{array}{l}\text { Español } \\
\text { España }\end{array}$ & $\begin{array}{l}\text { Evaluación formativa y compartida para la } \\
\text { inclusión de descansos activos en infantil }\end{array}$ \\
\hline $\begin{array}{l}\text { (Romero, Gómez, y } \\
\text { Pinzón, 2018) }\end{array}$ & Scopus & $\begin{array}{l}\text { Perfiles Educativos | } \\
\text { vol. XL, núm. } 162 .\end{array}$ & $\begin{array}{l}\text { Español } \\
\text { México }\end{array}$ & $\begin{array}{l}\text { Compartir metas de aprendizaje como } \\
\text { estrategia de evaluación formativa. Un caso } \\
\text { con profesores de matemáticas }\end{array}$ \\
\hline
\end{tabular}




\begin{tabular}{|c|c|c|c|c|}
\hline Autor(es). año & Base de datos & Revista & País/lengua & Nombre de la publicación \\
\hline (Díaz López, 2018) & Scopus & $\begin{array}{l}\text { Educación Médica } \\
\text { Superior vol.32 no.3 }\end{array}$ & $\begin{array}{l}\text { Español } \\
\text { Cuba }\end{array}$ & $\begin{array}{l}\text { Impacto de la retroalimentación y la } \\
\text { evaluación formativa en la ensenanza- } \\
\text { aprendizaje de Biociencias }\end{array}$ \\
\hline (Shavelson, 2018) & Scopus & $\begin{array}{l}\text { Education Policy } \\
\text { Analysis Archives, } \\
26(48)\end{array}$ & $\begin{array}{l}\text { Estados } \\
\text { Unidos. } \\
\text { Inglés }\end{array}$ & $\begin{array}{l}\text { Methodological perspectives: Standardized } \\
\text { (summative) or contextualized (formative) } \\
\text { evaluation? }\end{array}$ \\
\hline $\begin{array}{l}\text { (Martín y Herrero, } \\
\text { 2018) }\end{array}$ & Scopus & $\begin{array}{l}\text { Estud. pedagóg. } \\
\text { vol. } 44 \text { no. } 2\end{array}$ & $\begin{array}{l}\text { Chile } \\
\text { Español }\end{array}$ & $\begin{array}{l}\text { Evaluación formativa del lenguaje para } \\
\text { profesores de ciencias bilingües: un estudio } \\
\text { de caso }\end{array}$ \\
\hline $\begin{array}{l}\text { (Jiménez, Pastor, y } \\
\text { Obrador, 2018) }\end{array}$ & Scopus & $\begin{array}{l}\text { Estudios Pedagógicos } \\
\text { XLIV, } \mathrm{N}^{\circ} 2: 21-38\end{array}$ & $\begin{array}{l}\text { Chile } \\
\text { Español }\end{array}$ & $\begin{array}{l}\text { ¿Por qué Hago Evaluación Formativa en } \\
\text { Educación Física? Relato Autobiográfico de } \\
\text { un Docente }\end{array}$ \\
\hline (Contreras, 2018) & Scopus & $\begin{array}{l}\text { Formación } \\
\text { Universitaria, } 11 \text { (4), } \\
\text { pp. 83-94. }\end{array}$ & $\begin{array}{l}\text { Chile } \\
\text { Español }\end{array}$ & $\begin{array}{l}\text { Retroalimentación por Pares en la Docencia } \\
\text { Universitaria. Una Alternativa de Evaluación } \\
\text { Formativa }\end{array}$ \\
\hline $\begin{array}{l}\text { (Saiz-Linares y } \\
\text { Susinos-Rada, 2018) }\end{array}$ & Scopus & $\begin{array}{l}\text { Meta: Avaliacao } \\
\text { 10(30):53 }\end{array}$ & $\begin{array}{l}\text { Brasil } \\
\text { Español }\end{array}$ & $\begin{array}{l}\text { Los procesos de retroalimentacion y la } \\
\text { evaluación formativa en un "practicum" } \\
\text { reflexivo de maestro. }\end{array}$ \\
\hline (Hooker, 2017) & Sciendirect & $\begin{array}{l}\text { Teaching and } \\
\text { Teacher Education }\end{array}$ & $\begin{array}{l}\text { Nueva } \\
\text { Zelandia }\end{array}$ & $\begin{array}{l}\text { Transforming teachers' formative assessment } \\
\text { practices through ePortfolios }\end{array}$ \\
\hline $\begin{array}{l}\text { (Meusen-Beekman, } \\
\text { Joosten-ten Brinke, y } \\
\text { Boshuizen, 2016) }\end{array}$ & Sciendirect & $\begin{array}{l}\text { Studies in } \\
\text { Educational } \\
\text { Evaluation }\end{array}$ & Inglés & $\begin{array}{l}\text { Effects of formative assessments to develop } \\
\text { self-regulation among sixth grade students: } \\
\text { Results from a randomized controlled } \\
\text { intervention }\end{array}$ \\
\hline $\begin{array}{l}\text { (Pla-Campas, } \\
\text { Arumí-Prat, Senye- } \\
\text { Mir, y Ramírez, } \\
\text { 2016) }\end{array}$ & Sciendirect & $\begin{array}{l}\text { Procedia - Social and } \\
\text { Behavioral Sciences }\end{array}$ & Inglés & $\begin{array}{l}\text { Efecto del uso de técnicas de evaluación } \\
\text { formativa en las calificaciones de los } \\
\text { estudiantes }\end{array}$ \\
\hline $\begin{array}{l}\text { (Maier, Wolf, y } \\
\text { Randler, 2016) }\end{array}$ & Sciendirect & $\begin{array}{l}\text { Computers y } \\
\text { Education }\end{array}$ & $\begin{array}{l}\text { Alemania } \\
\text { Inglés }\end{array}$ & $\begin{array}{l}\text { Effects of a computer-assisted formative } \\
\text { assessment intervention based on multiple- } \\
\text { tier diagnostic items and different feedback } \\
\text { types }\end{array}$ \\
\hline $\begin{array}{l}\text { (Chavez Y.; Martinez } \\
\text { F., 2018) }\end{array}$ & Scielo & $\begin{array}{l}\text { Educación } \\
\text { Matemática, vol. 30, } \\
\text { núm. } 3\end{array}$ & $\begin{array}{l}\text { México } \\
\text { Español }\end{array}$ & $\begin{array}{l}\text { Evaluar para aprender: hacer más compleja la } \\
\text { tarea a los alumnos }\end{array}$ \\
\hline $\begin{array}{l}\text { (Hernández- } \\
\text { Elizondo y Salicetti- } \\
\text { Fonseca, 2018) }\end{array}$ & Scielo & $\begin{array}{l}\text { Estudios Pedagógicos } \\
\text { XLIV, } \mathrm{N}^{\circ} 2: 297-310\end{array}$ & $\begin{array}{l}\text { Costa Rica } \\
\text { Español }\end{array}$ & $\begin{array}{l}\text { La Evaluación Formativa en el Proceso } \\
\text { Enseñanza-aprendizaje en } \\
\text { Estudiantes de Actividad Deportiva de la } \\
\text { Universidad de Costa Rica }\end{array}$ \\
\hline
\end{tabular}




\begin{tabular}{|c|c|c|c|c|}
\hline Autor(es). año & Base de datos & Revista & País/lengua & Nombre de la publicación \\
\hline $\begin{array}{l}\text { (Gallardo-Fuentes, } \\
\text { López-Pastor, y } \\
\text { Carter-Thuillier, } \\
\text { 2020)(Gallardo- } \\
\text { Fuentes et al., 2020) }\end{array}$ & Scielo & $\begin{array}{l}\text { Estudios Pedagógicos } \\
\text { XLIV, } \mathrm{N}^{\circ} 2: \text { 55-77, }\end{array}$ & $\begin{array}{l}\text { España } \\
\text { Español }\end{array}$ & $\begin{array}{l}\text { Efectos de la Aplicación de un Sistema de } \\
\text { Evaluación Formativa en la Autopercepción } \\
\text { de Competencias Adquiridas en Formación } \\
\text { Inicial del Profesorado }\end{array}$ \\
\hline $\begin{array}{l}\text { (Sonlleva, Martínez, } \\
\text { y Monjas, 2018) }\end{array}$ & Scielo & $\begin{array}{l}\text { Estudios Pedagógicos } \\
\text { XLIV, } \mathrm{N}^{\circ} 2: 329-351\end{array}$ & $\begin{array}{l}\text { España } \\
\text { Español }\end{array}$ & $\begin{array}{l}\text { Los Procesos de Evaluación y sus } \\
\text { Consecuencias. Análisis de la Experiencia del } \\
\text { Profesorado de Educación Física }\end{array}$ \\
\hline $\begin{array}{l}\text { (Zhu, Liu, y Lee, } \\
2020)\end{array}$ & Sciendirect & $\begin{array}{l}\text { Computers y } \\
\text { Education }\end{array}$ & $\begin{array}{l}\text { Estados } \\
\text { Unidos } \\
\text { Inglés }\end{array}$ & $\begin{array}{l}\text { The effect of automated feedback on revision } \\
\text { behavior and learning gains in formative } \\
\text { assessment of scientific argument writing }\end{array}$ \\
\hline (van der Kleij, 2019) & Sciendirect & $\begin{array}{l}\text { Teaching and } \\
\text { Teacher Education }\end{array}$ & $\begin{array}{l}\text { Australia } \\
\text { Inglés }\end{array}$ & $\begin{array}{l}\text { Comparison of teacher and student } \\
\text { perceptions of formative assessment feedback } \\
\text { practices and association with individual } \\
\text { student characteristics }\end{array}$ \\
\hline $\begin{array}{l}\text { (Chang y Wimmers, } \\
\text { 2017) }\end{array}$ & Sciendirect & $\begin{array}{l}\text { Health Professions } \\
\text { Education3 (2017) } \\
\text { 32-37 }\end{array}$ & $\begin{array}{l}\text { Estados } \\
\text { Unidos } \\
\text { Inglés }\end{array}$ & $\begin{array}{l}\text { Effect of Repeated/Spaced Formative } \\
\text { Assessments on Medical School Final Exam } \\
\text { Performance }\end{array}$ \\
\hline $\begin{array}{l}\text { (Fives y Barnes, } \\
2020)\end{array}$ & Sciendirect & $\begin{array}{l}\text { Teaching and } \\
\text { Teacher Education }\end{array}$ & $\begin{array}{l}\text { Estados } \\
\text { Unidos } \\
\text { Inglés }\end{array}$ & $\begin{array}{l}\text { Navigating the complex cognitive task of } \\
\text { classroom assessment }\end{array}$ \\
\hline $\begin{array}{l}\text { (Martínez-Miguel, } \\
\text { Solano-Ruiz, García- } \\
\text { Carpintero Blas, y } \\
\text { Manso-Perea, 2018) }\end{array}$ & Scielo & Enfermería Global & $\begin{array}{l}\text { España } \\
\text { Español }\end{array}$ & $\begin{array}{l}\text { Impacto de la evaluación de competencias } \\
\text { en la calidad del aprendizaje: percepción de } \\
\text { discentes y docentes de Grado en Enfermería }\end{array}$ \\
\hline $\begin{array}{l}\text { (De Araujo Santos y } \\
\text { de Bonna, 2018) }\end{array}$ & Scielo & $\begin{array}{l}\text { Revista electrónica } \\
\text { de investigación en } \\
\text { educación ciencias }\end{array}$ & $\begin{array}{l}\text { Brasil } \\
\text { Portugués }\end{array}$ & $\begin{array}{l}\text { Entre provas e instruções: observando } \\
\text { a prática avaliativa dos professores de } \\
\text { matemática das Escolas de Referência da rede } \\
\text { estadual de Pernambuco }\end{array}$ \\
\hline $\begin{array}{l}\text { (Perazzi y Celman, } \\
\text { 2017) }\end{array}$ & Scielo & $\begin{array}{l}\text { Praxis educativa, Vol. } \\
\text { XXI, No } 3 \text {, }\end{array}$ & $\begin{array}{l}\text { Argentina } \\
\text { Español }\end{array}$ & $\begin{array}{l}\text { La evaluación de los aprendizajes en aulas } \\
\text { universitarias: una investigación sobre las } \\
\text { prácticas }\end{array}$ \\
\hline
\end{tabular}




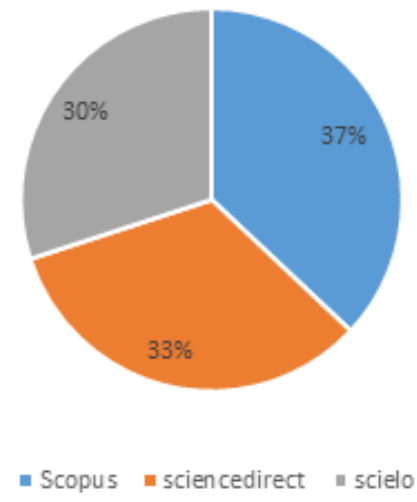

Figura 2. Porcentaje de artículos encontrados por fuente.

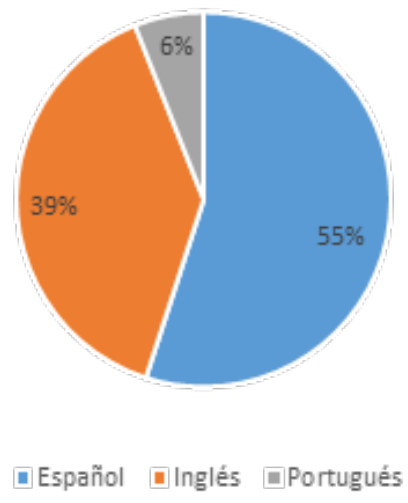

Figura 3. Porcentaje de artículos encontrados por idioma.

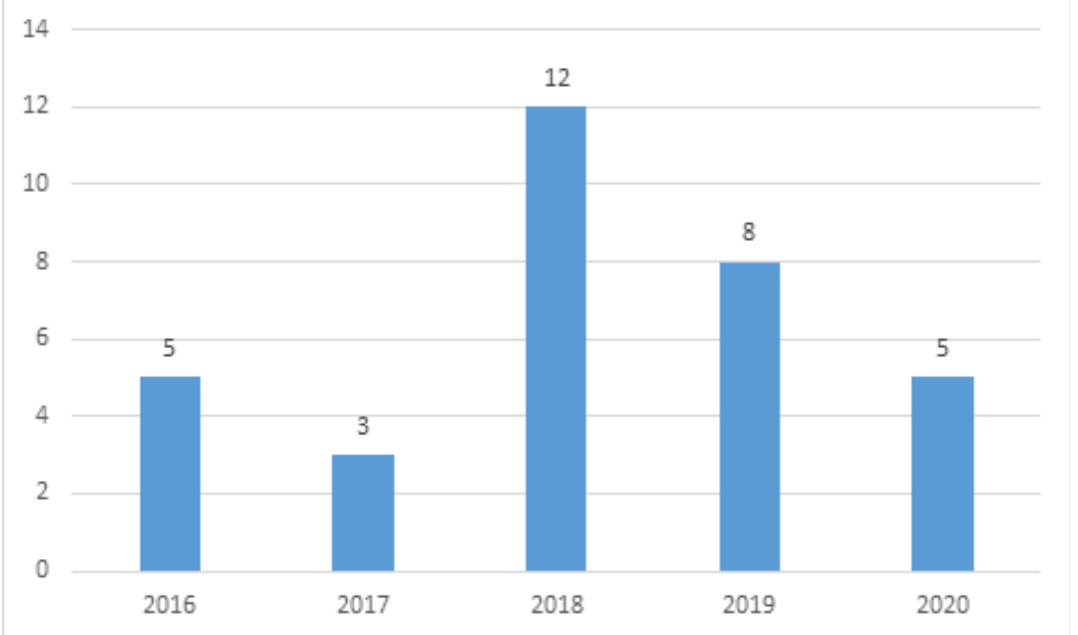

Figura 4. Número de artículos encontrados en los últimos 5 años. 
Se observa en la Figura 2, con respecto a la base de datos, se encontró que el 35\% corresponde a Scopus, 33\% a Sciencedirect y el 30\% a Scielo. En relación a la Figura 3, relacionado a los idiomas de publicación, se encontró que el 55\% está escrito en el idioma español, el 39\% en inglés y el 6\% en portugués, revista de diferentes países de los continentes de América, Asia, Europa y Oceanía.

Con respecto a las publicaciones de revistas en los últimos cinco años, se encontró 5 artículos en el año 2016 que equivale al 15\%, 3 artículos en 2017 que en porcentaje es el 9\%, 12 artículos en el año 2018 equivalente a $37 \%$ del total, 8 en 2019 lo que representa al 24\% y 5 en 2020 que representa el $15 \%$ del total de artículos revisados tal y como se evidencia en la Figura 4.

Además, se organizaron los datos en una matriz ( anexo 1) en la que se consideró la fuente, el objetivo, unidad de análisis o muestra de estudio, recogida de datos, instrumentos utilizados y las conclusiones a las que se llegó.

\section{Discusión}

Tomado en cuenta el anexo 1 , se coincide plenamente con las conclusiones a las que arribaron sobre la evaluación formativa en aula, dado que han generado buenos resultados en el aprendizaje (López Pastor et al, 2020; Angelini, 2016; Pascual-Arias et al., 2019; Martín y Herrero, 2018; Jiménez et al., 2018). Esto ha permitido tener mejor rendimiento en la materia desarrollada, pues el estudiante es capaz de intervenir en su proceso de aprendizaje, tomar sus propias decisiones lo cual se traduce en el desarrollo de la autonomía. Como afirma Rivers et al., (2019) el de regular su aprendizaje.

La evaluación formativa se realiza para valorar el proceso al reconocer las dificultades y las mejoras que ocurren durante el aprendizaje de los estudiantes. En el proceso de la autoevaluación y evaluación de pares, como en aspectos formales de la asignatura, hay dificultades, puesto que no hay una cultura de evaluación. Si bien existen normas y orientaciones para implementar la evaluación formativa en el aula, estas se aplican de forma esporádica o muy poco.

La retroalimentación es esencial en la evaluación, pues fomenta del desarrollo integral del estudiante, además que causa confianza, la autonomía; es participativa $y$, sobre todo, genera mejora de los niveles de aprendizaje, pues el estudiante adquiere confianza al recibir en forma oral o escrita las fortalezas, así como las dificultades de su evidencia, lo cual le permite mejorar y conlleva a un aprendizaje significativo.

Hoy, en la sociedad del conocimiento, existe mucha información, por tanto, la autoformación y el fortalecimiento de las capacidades del docente en la evaluación formativa es esencial para la mejora de los niveles de aprendizaje en los estudiantes.

Tradicionalmente, la evaluación se plantea en tres momentos: inicial, de proceso y final, lo que coincide con la evaluación diagnóstica, formativa y sumativa, respectivamente. La evaluación diagnóstica intenta explorar las competencias que ya poseen los alumnos al inicio de una situación educativa; a diferencia de la sumativa, que permite tener un juicio global del logro de cada alumno al concluir dicha situación.

\section{REFERENCIAS}

Angelini, L. (2016). Estudio sobre evaluación formativa y compartida en la formación docente en inglés. Actualidades Investigativas En Educación.

Bizarro, W., Sucari, W., y Quispe-Coaquira, A. (2019). Evaluación formativa en el marco del 
enfoque por competencias. Revista Innova Educación, 1. https://doi.org/https://doi. org/10.35622/j.rie.2019.02.008

Black, P., y Wiliam, D. (1998). Assessment and Classroom Learning. Assessment in Education: Principles, Policy y Practice (Vol. 5). https://doi. org/10.1080/0969595980050102

Breckler, S. J., y Wiggins, E. C. (1989). Affect versus evaluation in the structure of attitudes. Journal of Experimental Social Psychology, 25(3), 253-271. https://doi.org/10.1016/00221031(89)90022-X

Chang, E. K., y Wimmers, P. F. (2017). Effect of Repeated/Spaced Formative Assessments on Medical School Final Exam Performance. Health Professions Education, 3(1), 32-37. https://doi. org/10.1016/j.hpe.2016.08.001

Chavez Y.; Martinez F. (2018). Evaluar para aprender: hacer más compleja la tarea a los alumnos. Educacion Matematica (Vol. 30). https://doi.org/10.24844/EM3003.09

Contreras, G. A. (2018). Peer teacher feedback at the university. An alternative of formative assessment. Formacion Universitaria, 11(4), 83-94. https://doi.org/10.4067/S071850062018000400083

De Araujo Santos, A., y de Bonna, V. (2018). Entre provas e instruções: observando a prática avaliativa dos professores de matemática das Escolas de Referência da rede estadual de Pernambuco. Revista Electrónica de Investigación En Educación En Ciencias, 13(1), $01-11$.

Díaz López, M. M. (2018). The impact of feedback and formative evaluation on biosciences teaching-learning. Revista Cubana de Educacion Medica Superior, 32(3), 147-156.

Dochy, F., Segers, M., y Sluijsmans, D. (2012). Studies in Higher Education review $\mathrm{T}$ h e U s e of Self-, Peer and C o- a s s e s s m e $\mathrm{n} \mathrm{t} \mathrm{m}$ Higher Education: a review, (October 2012), $37-41$.
Fives, H., y Barnes, N. (2020). Navigating the complex cognitive task of classroom assessment. Teaching and Teacher Education, 92, 103063. https://doi.org/10.1016/j.tate.2020.103063

Gallardo-Fuentes, F. J., López-Pastor, V. M., y CarterThuillier, B. I. (2020). Advantages and Issues of Formative Assessment and its Influence on SelfPerception of Competences in students of Preservice Physical Education Teacher Training. Retos, 83, 417-424.

Gomez, L., Muñoz, M., y Londoño-Vásquez, D. (2019). Prácticas evaluativas en la escuela. Una ruta pedagógica hacia la construcción de aprendizajes significativos. Revista Ale- Theia, 11(1), 37-68, 37-68.

González Castillo, D. E., Varela Ruiz, M., y Fortoul van der Goes, T. I. (2016). El proceso de la evaluación formativa desde el modelo Weisbord en educación médica. Investigación En Educación Médica, 5(19), 136-147. https://doi. org/10.1016/j.riem.2015.11.002

Hattie, J. A. C. (2009). A synthesis of over 800 metaanalyses relating to achievement. Londres. Retrieved from http://people.oregonstate. edu/ flayb/MY COURSES/H676 MetaAnalysis Fall2016/Examples of SRs y MAs of interventions/Hattie2000 Synthesis of over 800 meta-analyses relating to achievement - first 29 pages.pdf

Hattie, J., y Timperley, H. (2007). The Power of Feedback. Review of Educational Research, 77(1), 81-112. https://doi. org/10.3102/003465430298487

Hernández-Elizondo, J., y Salicetti-Fonseca, A. (2018). La Evaluación Formativa en el Proceso Enseñanza-aprendizaje en Estudiantes de Actividad Deportiva de la Universidad de Costa Rica. Estudios Pedagógicos (Valdivia), 44(2), 297-310. https://doi.org/10.4067/s071807052018000200297 
Hooker, T. (2017). Transforming teachers' formative assessment practices through ePortfolios. Teaching and Teacher Education, 67, 440-453. https://doi.org/10.1016/j.tate.2017.07.004

Núñez-Peña, I M., Bono, R., y Suárez-Pellicioni, M. (2015). Evaluación Formativa en Educación Superior: Impacto en Estudiantes con Ansiedad a las Matemáticas. Procedia - Social and Behavioral Sciences, 196(July 2014), 135-141. https://doi.org/10.1016/j.sbspro.2015.07.023

Jiménez, T. C., Pastor, V. M. L., y Obrador, E. S. (2018). Why do i use formative evaluation in physical education? A eeacher's autobiographical account. Estudios Pedagogicos, 44(2), 21-38. https://doi. org/10.4067/S0718-07052018000200021

López-Benavente, A., Ureña-Ortín, N., y Alarcón López, F. (2019). Formative and shared assessment for the inclusion of active breaks in kindergarten. Journal of Sport and Health Research, 11(Supl 1), 143-154.

López-Pastor, V.; Sonllev.-V. M., y Suyapa-Martínez, S. (2019). Evaluación Formativa y Compartida en Educación Formative and Shared Evaluation in Education. Revista Iberoamericana de Evaluación Educativa, 12(1), 5-9. Retrieved from www.rinace.net/riee/https://revistas.uam. es/riee

López-Pastor V. (2017). Buenas prácticas docentes: evaluación formativa (Grupo IFAH). Ciudad de Léon: 2017. Retrieved from file:///C:/ Users/USUARIO/Downloads/Libro ULE BuenasPrácticasDocentesLópez-PastoryPérezPueyocoord.2017.pdf

López Pastor, V. M., Soria, M. M., Arias, C. P., y Arribas, J. C. M. (2020). The importance of using Formative and Shared Assessment in Physical Education Teacher Education: Tutored Learning Projects as an example of good practice. Retos, 37, 620-627.

Maier, U., Wolf, N., y Randler, C. (2016). Effects of a computer-assisted formative assessment intervention based on multiple-tier diagnostic items and different feedback types. Computers and Education, 95, 85-98. https://doi. org/10.1016/j.compedu.2015.12.002

Martín, M., y Herrero, M. (2018). Evaluación Formativa de la Lengua de los Profesores de Science: un Estudio de Caso. Estudios Pedagógicos (Valdivia), 44(2), 279-296. https:// doi.org/10.4067/s0718-07052018000200279

Martínez-Miguel, E., Solano-Ruiz, M. del C., GarcíaCarpintero Blas, E., y Manso-Perea, C. (2018). Impacto de la evaluación de competencias en la calidad del aprendizaje: percepción de discentes y docentes de Grado en Enfermería TT Competency assessment impact in quality of learning: Nursing degree learner's and teacher's perception. Enfermería Global, 17(50), 400-429. https://doi.org/10.6018/eglobal.17.2.263041

Martínez-Rizo, F., y Mercado, A. (2015). Estudios sobre prácticas de evaluación en el aula: Revisión de la literatura. Revista Electronica de Investigacion Educativa, 17(1), 17-32.

Meusen-Beekman, K. D., Joosten-ten Brinke, D., y Boshuizen, H. P. A. (2016). Effects of formative assessments to develop self-regulation among sixth grade students: Results from a randomized controlled intervention. Studies in Educational Evaluation, 51, 126-136. https:// doi.org/10.1016/j.stueduc.2016.10.008

Mohamadi, Z. (2018). Studies in Educational Evaluation Comparative e ff ect of online summative and formative assessment on EFL student writing ability. Studies in Educational Evaluation, 59(February), 29-40. https://doi. org/10.1016/j.stueduc.2018.02.003

Ocaña A., G., Pulido, L., y Gil, S. (2019). Changes in the performance of students of a mathematical thinking course through formative assessment with an online question bank. Interdisciplinaria, 36(1), 7-22.

Pascual-Arias, C., García-Herranz, S., y LópezPastor, V. M. (2019). What do preschool students want? The role of formative and shared 
assessment in their right to decide / ¿Qué quieren los niños y niñas de Educación Infantil? El papel de la evaluación formativa y compartida en su derecho a decidir. Cultura y Educacion, 31(4), 865-880. https://doi.org/10.1080/11356405.201 9.1656486

Perazzi, M., y Celman, S. (2017). The evaluation of learning in university classrooms: a research on the practices. Praxis Educativa, 21(3), 23-31. https://doi.org/10.19137/ praxiseducativa-2017-210303

Pla-Campas, G., Arumí-Prat, J., Senye-Mir, A. M., y Ramírez, E. (2016). Effect of Using Formative Assessment Techniques on Students' Grades. Procedia - Social and Behavioral Sciences, 228(June), 190-195. https://doi.org/10.1016/j. sbspro.2016.07.028

Popham J. (2013). Evaluación trans-formativa_El poder transformador de la evaluación formativa (Edicones N). Madrid: 2013.

Proust, M., Stiggins, R. J. (2002). A real voyage of discovery consists not of seeking new landscapes but of seeing through new eyes. A SPECIAL SECTION ON ASSESSMENT. Retrieved from http://journals.sagepub.com/ doi/pdf/10.1177/003172170208301010

Ravela, P. L. (2017). ¿Cómo mejorar la Evaluación en el aula?

Ravela P. y Cardoner M. (2019). Transformando las prácticas de evaluación a través del trabajo colaborativo. (G. M. Editores, Ed.). Montevideo.

Rivers, M. L., Dunlosky, J., y Joynes, R. (2019). The contribution of classroom exams to formative evaluation of concept-level knowledge. Contemporary Educational Psychology, 59(October), 101806. https://doi.org/10.1016/j. cedpsych.2019.101806

Romero, I., Gómez, P., y Pinzón, A. (2018). Compartir metas de aprendizaje como estrategia de evaluación formativa: Un caso con profesores de matemáticas. Perfiles Educativos, 40(162), 117-137. https://doi.org/10.22201/ iisue.24486167e.2018.162.58632
Saiz-Linares, A., y Susinos-Rada, T. (2018). Feedback and formative evaluation in a teachers reflexive practicum. Meta: Avaliacao, 10(30), 533-554. https://doi.org/10.22347/2175-2753v10i30.1605

Santos, L. (2016). A articulação entre a avaliação somativa e a formativa, na prática pedagógica: Uma impossibilidade ou um desafio? Ensaio, 24(92), 637-669. https://doi.org/10.1590/ S0104-40362016000300006

Schildkamp, K., van der Kleij, F. M., Heitink, M. C., Kippers, W. B., y Veldkamp, B. P. (2020). Formative assessment: A systematic review of critical teacher prerequisites for classroom practice. International Journal of Educational Research, 103(April), 101602. https://doi. org/10.1016/j.ijer.2020.101602

Shavelson, R. J. (2018). Methodological perspectives: Standardized (summative) or contextualized (formative) evaluation? Education Policy Analysis Archives, 26, 2020. https://doi. org/10.14507/epaa.26.3813

Shute, V. J. (2008). Focus on Formative Feedback. Review of Educational Research, 78(1), 153189. https://doi.org/10.3102/0034654307313795

Sonlleva, M., Martínez, S., y Monjas, R. (2018). Los Procesos de Evaluación y sus Consecuencias. Análisis de la Experiencia del Profesorado de los Procesos de Evaluación y sus Consecuencias. Análisis de la Experiencia del Profesorado de Educación Física. Estudios Pedagógicos XLIV, $\mathrm{N}^{\circ}, 2,329-351$.

Talanquer, V. (2015). The importance of formative assessment. Educacion Quimica, 26(3), 177179. https://doi.org/10.1016/j.eq.2015.05.001

Tunstall P., y Gipps C. (1996). Teacher feedback to young children in formative assessment: a typology. British Educational Research Journal, 22(4), 389-404.

Van der Kleij, F. M. (2019). Comparison of teacher and student perceptions of formative assessment feedback practices and association with individual student characteristics. Teaching and 
Teacher Education, 85, 175-189. https://doi. org/10.1016/j.tate.2019.06.010

Wiggins, G. (1989). The futility of trying to teach everything of importance. Educational Leadership, 47(3), 44-48,57-59.

Xiaotong; Chen Wujin; Liao Libin; Li Tian; Qin Wen y Bai. (2019). Analysis of the teaching effect of the normal human morphology with mixed teaching mode and formative evaluation in china [ Article @ Análisis del efecto de la enseñanza de morfología humana normal con un modelo de enseñanza mixta y evaluación formativa e. https://doi.org/10.4067/S071795022019000301085
Yin, X., y Buck, G. A. (2019). Using a collaborative action research approach to negotiate an understanding of formative assessment in an era of accountability testing. Teaching and Teacher Education, 80, 27-38. https://doi.org/10.1016/j. tate.2018.12.018

Zhu, M., Liu, O. L., y Lee, H. S. (2020). The effect of automated feedback on revision behavior and learning gains in formative assessment of scientific argument writing. Computers and Education, 143(September 2018), 103668. https://doi.org/10.1016/j.compedu.2019.103668 
Anexo 1. Características principales de resumen de los artículos.

\begin{tabular}{|c|c|c|c|c|}
\hline Fuente & Objetivo o propósito & Unidad de análisis o muestra & Recogida de datos/Instrumentos & Resultados, conclusiones \\
\hline $\begin{array}{l}\text { (Gómez, L.; Muñoz } \\
\text { M, Et al, 2019) }\end{array}$ & $\begin{array}{l}\text { Analizar las prácticas evaluativas } \\
\text { de los docentes posibilita la } \\
\text { construcción significativa de } \\
\text { aprendizajes. }\end{array}$ & $\begin{array}{l}\text { Cuatro madres de familia, dos } \\
\text { directivos y dos docentes; en } \\
\text { dos instituciones educativas. }\end{array}$ & $\begin{array}{l}\text { Se aplicaron entrevistas, } \\
\text { observaciones de clase y talleres } \\
\text { didácticos. }\end{array}$ & $\begin{array}{l}\text { Los docentes las encuentran en factores externos } \\
\text { sobre la evaluación formativa. }\end{array}$ \\
\hline $\begin{array}{l}\text { (López Pastor et al., } \\
\text { 2020) }\end{array}$ & $\begin{array}{l}\text { Analizar la importancia de utilizar } \\
\text { procesos de evaluación formativa y } \\
\text { compartida. }\end{array}$ & $\begin{array}{l}\text { Estudiantes de formación } \\
\text { inicial docente. }\end{array}$ & $\begin{array}{l}\text { Ficha de autoevaluación reflexiva } \\
\text { sobre la sesión de la puesta } \\
\text { en práctica del Proyecto de } \\
\text { Aprendizaje Tutorado (PAT). }\end{array}$ & $\begin{array}{l}\text { Ha generado buenos resultados en el aprendizaje, la } \\
\text { satisfacción y la autopercepción de las habilidades } \\
\text { de enseñanza. }\end{array}$ \\
\hline $\begin{array}{l}\text { (Pascual-Arias et al., } \\
\text { 2019) }\end{array}$ & $\begin{array}{l}\text { Fomentar el derecho a participar y } \\
\text { decidir del alumnado en su propia } \\
\text { educación a través de este tipo de } \\
\text { evaluación. }\end{array}$ & $\begin{array}{l}\text { Niños y niñas de Educación } \\
\text { Infantil. }\end{array}$ & $\begin{array}{l}\text { Observación participante. } \\
\text { Entrevistas grupales } \\
\text { semiestructuradas. Lecciones } \\
\text { significativas }\end{array}$ & $\begin{array}{l}\text { El alumnado es capaz de expresar y proponer } \\
\text { cambios en su aula, tomar decisiones, aportar ideas } \\
\text { sobre el proceso de enseñanza-aprendizaje. }\end{array}$ \\
\hline (Rivers et al., 2019) & $\begin{array}{l}\text { Monitorear que los estudiantes } \\
\text { pueden participar durante un } \\
\text { examen de clase. }\end{array}$ & $\begin{array}{l}403 \text { estudiantes de pregrado (la } \\
\text { mayoría entre las edades de } 18 \\
\text { a } 21 \text { años. }\end{array}$ & Cuestionario & $\begin{array}{l}\text { La evaluación formativa ayuda a los estudiantes } \\
\text { regular su aprendizaje. }\end{array}$ \\
\hline $\begin{array}{l}\text { (Schildkamp et al., } \\
\text { 2020) }\end{array}$ & $\begin{array}{l}\text { ¿Qué requisitos previos de los } \\
\text { docentes deben existir para utilizar } \\
\text { la evaluación formativa en su aula? }\end{array}$ & 54 estudios & Revisiones sistemáticas de literatura & $\begin{array}{l}\text { El papel del profesor es muy importante en el uso de } \\
\text { la evaluación formativa en aula. }\end{array}$ \\
\hline (Yin y Buck, 2019) & $\begin{array}{l}\text { Implementar la formativa evaluación } \\
\text { en el contexto de las pruebas de } \\
\text { responsabilidad acumulativa en los } \\
\text { EE. UU. }\end{array}$ & $\begin{array}{l}30 \text { estudiantes en cada sección, } \\
\text { en } 03 \text { grupos }\end{array}$ & $\begin{array}{l}\text { Se realizó en un aula de ciencias, } \\
\text { investigación acción colaborativa }\end{array}$ & $\begin{array}{l}\text { Identificaron seis temas: (1) negociar objetivos de } \\
\text { aprendizaje, (2) reestructurar la instrucción, (3) } \\
\text { implementar estrategias efectivas de evaluación } \\
\text { (4) negociación de limitaciones de tiempo, (5) } \\
\text { modificación de pruebas sumativas y (6) negociación } \\
\text { con estudiantes. }\end{array}$ \\
\hline
\end{tabular}




\begin{tabular}{|c|c|c|c|c|}
\hline Fuente & Objetivo o propósito & Unidad de análisis o muestra & Recogida de datos/Instrumentos & Resultados, conclusiones \\
\hline $\begin{array}{l}\text { (Xiaotong; Chen } \\
\text { Wujin; Liao Libin; Li } \\
\text { Tian; Qin Wen y Bai, } \\
\text { 2019) }\end{array}$ & $\begin{array}{l}\text { El uso de formativo } \\
\text { La evaluación en el proceso de } \\
\text { enseñanza }\end{array}$ & 15 clases & Cuestionario & $\begin{array}{l}\text { La evaluación en el proceso de enseñanza puede } \\
\text { ayudar a los instructores comprender la dinámica } \\
\text { del conocimiento de los estudiantes y si los métodos } \\
\text { de enseñanza específicos se usan correctamente. }\end{array}$ \\
\hline (Angelini, 2016) & $\begin{array}{l}\text { Evaluar y analizar los resultados de } \\
\text { la puesta en práctica de la evaluación } \\
\text { formativa y compartida. }\end{array}$ & 135 estudiantes & $\begin{array}{l}\text { Entrevistas personalizadas por } \\
\text { equipos e individuales. }\end{array}$ & $\begin{array}{l}\text { Permite un mejor rendimiento académico. } \\
\text { Se encuentran inseguridades del alumnado } \\
\text { frente a la propuesta de autoevaluación y } \\
\text { evaluación de pares como a aspectos formales. }\end{array}$ \\
\hline (Santos, 2016) & $\begin{array}{l}\text { La articulación entre evaluación } \\
\text { sumativa y formativa, entendida } \\
\text { aquí como complementariedad }\end{array}$ & 169 estudiantes & $\begin{array}{l}\text { El cuaderno diario. El portafolio. } \\
\text { Cuestionario y entrevistas } \\
\text { semiestructuradas Observación de } \\
\text { sesiones de trabajo colaborativo. }\end{array}$ & $\begin{array}{l}\text { Evaluación formativa, aunque recomendada } \\
\text { por los documentos. En cuanto al currículum e } \\
\text { investigación, ocurre esporádicamente en el aula. }\end{array}$ \\
\hline $\begin{array}{l}\text { (Ocaña A. et al., } \\
\text { 2019) }\end{array}$ & $\begin{array}{l}\text { Identificar características cualitativas } \\
\text { que han permitido realizar ajustes y } \\
\text { mejoras a la base de preguntas. }\end{array}$ & 27 Grupos de taller & $\begin{array}{l}\text { Encuestas, entrevistas y grupos } \\
\text { focales }\end{array}$ & $\begin{array}{l}\text { Mejoraeneldesempeñoyapropiación delosconceptos } \\
\text { matemáticosen aquellos estudiantes queacceden alos } \\
\text { exámenes en línea frente a aquellos que no los usan. }\end{array}$ \\
\hline $\begin{array}{l}\text { (López-Benavente et } \\
\text { al., 2019) }\end{array}$ & $\begin{array}{l}\text { Evaluar la eficacia de un programa } \\
\text { de educación física en la etapa de } \\
\text { infantil a través de un sistema de } \\
\text { evaluación formativa y compartida. }\end{array}$ & 46 niños, 5 a 6 años & $\begin{array}{l}\text { Estudio de caso, Diario Cuñas } \\
\text { Motrices }\end{array}$ & $\begin{array}{l}\text { Evaluación formativa y compartida son la clave } \\
\text { para implicar al alumnado en la evaluación de la } \\
\text { enseñanza, permitiendo al docente reflexionar } \\
\text { sobre su acción educativa, fomenta el diálogo } \\
\text { entre iguales y entre el profesor y sus alumnos. }\end{array}$ \\
\hline (Romero et al., 2018) & $\begin{array}{l}\text { Compartir las metas de aprendizaje } \\
\text { con el alumnado, que promueve la } \\
\text { autoevaluación de los estudiantes } \\
\text { y permite al profesor percibir su } \\
\text { progreso y adaptar su enseñanza. }\end{array}$ & 6 grupos de 4 profesores que & Entrevistas & $\begin{array}{l}\text { Promueve una comunicación auténtica en torno } \\
\text { al proceso de enseñanza-aprendizaje, implica } \\
\text { al alumnado en dicho proceso y orientar en } \\
\text { tiempo real la enseñanza de los profesores. }\end{array}$ \\
\hline
\end{tabular}

Horizontes. Revista de Investigación en Ciencia de la Educación 


\begin{tabular}{|c|c|c|c|c|}
\hline Fuente & Objetivo o propósito & Unidad de análisis o muestra & Recogida de datos/Instrumentos & Resultados, conclusiones \\
\hline
\end{tabular}

Díaz López, 2018) Obtener información acerca de las percepciones, las actitudes, las experiencias y las expectativas en relación con la evaluación formativa y el impacto de la retroalimentación a estudiantes.

(Shavelson, 2018) La evaluación educativa debiera basarse en evidencia estandarizada (sumativa, a menudo cuantitativa) o contextualizada (formativa a menudo cualitativa).

(Shavelson, 2018) (Martín y Herrero, 2018)

Proponer la evaluación formativa por medio de la observación entre iguales como una posible herramienta para contribuir a la formación del profesorado de educación bilingüe.

(Jiménez et al., 2018) Describe el proceso de evolución de un docente de educación física desde la perspectiva de los sistemas de evaluación que ha vivido como alumno y que ha utilizado como maestro.
7 participantes

Técnica de los grupos focales.

Grabación en audios.

Docentes y estudiantes

Preguntas:

1) ¿qué está pasando?

2) ¿hay un efecto sistemático?

3) De procesos o mecanismos:

Por qué o cómo pasa lo que está

pasando.

25 alumnos 7 a 8 años

Observación entre pares.

El papel del investigador.

Relato autobiográfico
Se evidenciaron 4 categorías que abarcan la comprensión del estudiante respecto de la función formativa de la evaluación, el empoderamiento de los estudiantes frente al proceso de evaluación formativa, el manejo de conflictos derivados de la evaluación y algunas sugerencias para mejorar los procesos de evaluación desde la perspectiva del estudiante.

Tres preguntas generales conducen la investigación y la evaluación: 1) Descriptiva - ¿qué está pasando? 2) Causal, ¿hay un efecto sistemático? 3) De procesos o mecanismos: Por qué o cómo pasa lo que está pasando. Igualmente, importantes son las políticas, los métodos e instrumentos de medición y los diseños al conducir las evaluaciones. Ignorarlas es asumir fuertes riesgos. La evaluación debe ser sensible al contexto. La política, los métodos de medición y el modelado son

Los resultados de la observación permiten ver el tipo de apoyo más frecuente y orientar al profesor en la mejora de su práctica.

A lo largo del relato, se puede observar cómo pasa de un modelo de evaluación sumativa y calificativa, fruto de su experiencia como estudiante y su formación inicial, hacia un modelo que toma como base la evaluación formativa y la práctica reflexiva. 


\begin{tabular}{|c|c|c|c|c|}
\hline Fuente & Objetivo o propósito & Unidad de análisis o muestra & Recogida de datos/Instrumentos & Resultados, conclusiones \\
\hline (Contreras, 2018) & $\begin{array}{l}\text { Ofrecer orientaciones teóricas y } \\
\text { metodológicas para implementar } \\
\text { la retroalimentación de la docencia } \\
\text { por pares en la educación superior. }\end{array}$ & $\begin{array}{l}28 \text { alumnos al principio y } 35 \\
\text { al final. }\end{array}$ & $\begin{array}{l}\text { Reunión de preobservación, registro } \\
\text { de la observación de clases, informe } \\
\text { previo, reunión de post observación } \\
\text { e informe final. }\end{array}$ & $\begin{array}{l}\text { La retroalimentación puede ser una poderosa } \\
\text { herramienta para mejorar la práctica docente } \\
\text { aunque debe contarse con una política instituciona } \\
\text { que la apoye. }\end{array}$ \\
\hline $\begin{array}{l}\text { (Saiz-Linares y } \\
\text { Susinos-Rada, 2018) }\end{array}$ & $\begin{array}{l}\text { Describir una propuesta formativa de } \\
\text { practicum que utiliza metodologías } \\
\text { didácticas activas y una evaluación } \\
\text { formativa. }\end{array}$ & Grupo de cinco alumnas & estudio de caso evaluativo & $\begin{array}{l}\text { Nueva manera de entender la evaluación en la } \\
\text { educación superior, identificando una serie de } \\
\text { beneficios que se desprenden de este tipo de } \\
\text { evaluación, así como algunas de las barreras que } \\
\text { podrían estar dificultando su desarrollo. }\end{array}$ \\
\hline Hooker, 2017 & $\begin{array}{l}\text { El uso de ePortfolios en un entorno } \\
\text { de ECE, cómo cambiaron las } \\
\text { prácticas de evaluación formativa de } \\
\text { los maestros mediante el uso de esta } \\
\text { tecnología. }\end{array}$ & $\begin{array}{l}\text { Profesores, niños y padres de } \\
\text { familia. }\end{array}$ & $\begin{array}{l}\text { Estudio de caso. Encuestas } \\
\text { semiestructuradas, entrevistas y } \\
\text { observaciones. }\end{array}$ & $\begin{array}{l}\text { Los ePortfolios brindan fácil acceso a ellos para } \\
\text { padres, familiares e hijos. }\end{array}$ \\
\hline $\begin{array}{l}\text { (Meusen-Beekman } \\
\text { et al., 2016) }\end{array}$ & $\begin{array}{l}\text { Examinamos si las evaluaciones } \\
\text { formativas mejorarían en cuanto } \\
\text { a la autorregulación, motivación y } \\
\text { autoeficacia entre los estudiantes de } \\
\text { sexto grado. }\end{array}$ & 695 participantes & $\begin{array}{l}\text { Intervención de evaluación } \\
\text { por pares, intervención de } \\
\text { autoevaluación o un control, } \\
\text { condición. }\end{array}$ & $\begin{array}{l}\text { No se encontraron diferencias significativas } \\
\text { entre la intervención de evaluación por pare } \\
\text { y la autoevaluación intervención en materia de } \\
\text { autorregulación, motivación o autoeficacia }\end{array}$ \\
\hline $\begin{array}{l}\text { (Pla-Campas et al., } \\
\text { 2016) }\end{array}$ & $\begin{array}{l}\text { El documento describe y discute el } \\
\text { Impacto de las técnicas de evaluación } \\
\text { participativa y no participativa } \\
\text { en el logro de los resultados del } \\
\text { aprendizaje. }\end{array}$ & $\begin{array}{l}118 \text { participantes de formación } \\
\text { docente }\end{array}$ & $\begin{array}{l}\text { Aplicación de técnicas la } \\
\text { autoevaluación, } \\
\text { evaluación por pares o evaluación } \\
\text { democrática. }\end{array}$ & $\begin{array}{l}\text { Las técnicas que se utilizaron en los sujetos pueder } \\
\text { haber influido en los resultados. }\end{array}$ \\
\hline
\end{tabular}




\begin{tabular}{|c|c|c|c|c|}
\hline Fuente & Objetivo o propósito & Unidad de análisis o muestra & Recogida de datos/Instrumentos & Resultados, conclusiones \\
\hline (Maier et al., 2016) & $\begin{array}{l}\text { Diagnosticar la comprensión } \\
\text { conceptual de los estudiantes } \\
\text { en dominios de aprendizaje con } \\
\text { conocimiento declarativo, en las } \\
\text { evaluaciones formativas asistidas } \\
\text { por computadora. }\end{array}$ & $\begin{array}{l}\text { Se utilizaron diez aulas de } \\
\text { secundaria y } 261 \text { alumnos } \\
\text { participaron en este estudio. }\end{array}$ & $\begin{array}{l}\text { Pruebas formativas asistidas por } \\
\text { ordenador. }\end{array}$ & $\begin{array}{l}\text { La comprensión, cuando los estudiantes usaron } \\
\text { la retroalimentación, fue superior a la ausencia de } \\
\text { retroalimentación y retroalimentación elaborada } \\
\text { cuando los estudiantes no la utilizaron. }\end{array}$ \\
\hline $\begin{array}{l}\text { (Chavez Y.; Martinez } \\
\text { F., 2018) }\end{array}$ & $\begin{array}{l}\text { Caracterizar las prácticas de } \\
\text { enseñanza-evaluación a partir de } \\
\text { la exigencia cognitiva de las tareas } \\
\text { que proponen los profesores a sus } \\
\text { alumnos para enseñar un contenido } \\
\text { matemático. }\end{array}$ & $\begin{array}{l}\text { Dos maestros de escuelas } \\
\text { primarias en Aguascalientes. } \\
\text { México }\end{array}$ & $\begin{array}{l}\text { Observación y videograbación } 12 \\
\text { clases de matemática }\end{array}$ & $\begin{array}{l}\text { La observación de las prácticas mostró que, aunque } \\
\text { las tareas propuestas a los estudiantes con base en } \\
\text { libros de texto y otros materiales tengan un alto } \\
\text { potencial matemático, es difícil para los docentes } \\
\text { mantener altos niveles de exigencia cognitiva } \\
\text { durante el desarrollo de las clases }\end{array}$ \\
\hline $\begin{array}{l}\text { (Hernández- } \\
\text { Elizondo y Salicetti- } \\
\text { Fonseca, 2018) }\end{array}$ & $\begin{array}{l}\text { Emplear la evaluación formativa } \\
\text { para valorar los aprendizajes por } \\
\text { parte del estudiantado que participa } \\
\text { de la clase de actividad deportiva. }\end{array}$ & $\begin{array}{l}\text { Ochenta estudiantes } \\
\text { universitarios }\end{array}$ & Cuestionario de autoreporte. & $\begin{array}{l}\text { Los beneficios percibidos por parte del estudiantado } \\
\text { reflejan la importancia de estos cursos dentro del } \\
\text { currículo universitario del ciclo formativo }\end{array}$ \\
\hline $\begin{array}{l}\text { (Gallardo-Fuentes et } \\
\text { al., 2020) }\end{array}$ & $\begin{array}{l}\text { Analizar la valoración del } \\
\text { alumnado sobre el sistema de } \\
\text { evaluación formativa y compartida } \\
\text { (EFyC) utilizado en la asignatura } \\
\text { "Fundamentos y didáctica de la } \\
\text { Educación corporal" }\end{array}$ & $\begin{array}{l}93 \text { alumnos, de dos grupos y } \\
\text { cursos }\end{array}$ & $\begin{array}{l}\text { Cuestionario de evaluación sobre } \\
\text { la asignatura y escala para la } \\
\text { autopercepción de competencia. }\end{array}$ & $\begin{array}{l}\text { Los resultados muestran una alta valoración de las } \\
\text { "ventajas" del sistema de EFyC utilizado. }\end{array}$ \\
\hline
\end{tabular}




\begin{tabular}{|c|c|}
\hline Fuente & Objetivo o propósito \\
\hline $\begin{array}{l}\text { (Sonlleva et al., } \\
2018 \text { ) }\end{array}$ & $\begin{array}{l}\text { Analizar sus vivencias ante este } \\
\text { hecho, a través del análisis de los } \\
\text { sentimientos y emociones que } \\
\text { genera la evaluación entre los } \\
\text { estudiantes, las interacciones entre } \\
\text { profesores y alumnos en el proceso } \\
\text { de evaluación. }\end{array}$ \\
\hline
\end{tabular}

(Zhu et al., 2020)

Explorar las revisiones habilitadas por el sistema de retroalimentación formativa se correlacionan con el desempeño del estudiante $y$ ganancias de aprendizaje
Unidad de análisis o muestra

Recogida de datos/Instrumentos
491 profesores de 17

universidades españolas

374 estudiantes de séptimo a duodécimo grado de 22 clases
Encuesta y 12 entrevistas personales Los resultados nos permiten afirmar que los profesionales de EF sienten ansiedad y temor hacia la evaluación; consideran fundamental la relación entre profesores y estudiantes para mejorar su proceso formativo; y afirman que el conocimiento previo del sistema de evaluación y su acuerdo son aspectos básicos para la motivación del aprendizaje y la reducción de los síntomas de tensión
Los archivos de registro del módulo climático.

\section{9 profesores y 186 estudiantes La encuesta.}

de inglés secundario y clases de

matemáticas en cinco escuelas

australianas retroalimentación entre profesores y estudiantes y (2) exploró la asociación entre las características individuales de los estudiantes y las percepciones de retroalimentación

(Chang y Wimmers, Las evaluaciones formativas, 2017) especialmente las espaciadas, fomentan hábitos de estudio efectivos, como la repetición de pruebas
Grupos por evaluación semanal formativa de desempeño y uso de exámenes prácticos /actuación. Examen final de práctica, múltiples
comparaciones utilizando

ANCOVA
Los estudiantes con mayores puntajes en promedio tenían más probabilidades de revisarse después de la retroalimentación automática, (2) las revisiones fueron positivamente relacionadas con los aumentos de puntaje, y (3) la retroalimentación contextualizada fue más efectiva para ayudar al aprendizaje

La calidad de los comentarios se percibió de manera más positiva por profesores que por estudiantes, y los profesores de inglés informaron niveles más altos de facilitación del uso de retroalimentación que estudiantes

El desempeño en las evaluaciones formativas semanales fue predictivo de las puntuaciones de los exámenes finales. Beneficiarse de los exámenes de prácticas extraacumulativas mientras que los estudiantes que son excelentes no necesitan examinar la práctica. 


\begin{tabular}{|c|c|c|c|c|}
\hline Fuente & Objetivo o propósito & Unidad de análisis o muestra & Recogida de datos/Instrumentos & Resultados, conclusiones \\
\hline $\begin{array}{l}\text { (Fives y Barnes, } \\
\text { 2020) }\end{array}$ & $\begin{array}{l}\text { Investigamos cómo un maestro de } \\
\text { alfabetización de quinto grado con } \\
\text { experiencia en evaluación de aula } \\
\text { integró evaluación en su práctica } \\
\text { docente. }\end{array}$ & Docentes de aula & $\begin{array}{l}\text { Estudio de caso cualitativo } \\
\text { intrínseco instrumental. }\end{array}$ & $\begin{array}{l}\text { Identificamos un repertorio de rutinas de gestión } \\
\text { e interpretación relevantes para la evaluación } \\
\text { estrategias que apoyaron su práctica de evaluación y } \\
\text { toma de decisiones educativas. }\end{array}$ \\
\hline $\begin{array}{l}\text { (Martínez-Miguel et } \\
\text { al., 2018) }\end{array}$ & $\begin{array}{l}\text { Conocer la percepción de discentes } \\
\text { y docentes en relación a las } \\
\text { prácticas actuales de evaluación de } \\
\text { competencias y su impacto en la } \\
\text { calidad del aprendizaje }\end{array}$ & $\begin{array}{l}\text { Para cuestionarios abiertos } \\
\text { fue de } 44 \text {, correspondiendo a } \\
30 \text { estudiantes y } 14 \text { docentes, } \\
\text { y de } 15 \text { para los grupos de } \\
\text { discusión, } 7 \text { estudiantes y } 8 \\
\text { docentes. }\end{array}$ & $\begin{array}{l}\text { Cuestionarios abiertos y grupos de } \\
\text { discusión de discentes y docentes }\end{array}$ & $\begin{array}{l}\text { Los docentes perciben dificultades para superar la } \\
\text { orientación tradicional de la evaluación, mientras } \\
\text { los estudiantes perciben su función más formativa } \\
\text { y demandan para ella un feedback suficiente y de } \\
\text { calidad. }\end{array}$ \\
\hline $\begin{array}{l}\text { (De Araujo Santos y } \\
\text { de Bonna, 2018) }\end{array}$ & $\begin{array}{l}\text { Analizar la práctica evaluativa de } \\
\text { los profesores de matemáticas de } \\
\text { EREM (Escuelas de Referencia en } \\
\text { Educación Secundaria) de la red del } \\
\text { estado de Pernambuco. }\end{array}$ & $\begin{array}{l}\text { Profe. GP 1: } 8 \text { observaciones, } \\
16 \text { lecciones. } 13 \text { horas y } \\
33 \text { minutos. Profe. GP } 2: 7 \\
\text { observaciones, } 14 \text { lecciones. } 11 \\
\text { horas y } 27 \text { minutos. }\end{array}$ & $\begin{array}{l}\text { Observación en dos clases del } \\
\text { primer año de secundaria. uso } \\
\text { de formulario de observación y } \\
\text { cuaderno de campo }\end{array}$ & $\begin{array}{l}\text { Predomina el carácter sumativo, siendo necesaria } \\
\text { una revisión en la propuesta de capacitación } \\
\text { permanente y acompañamiento de las actividades } \\
\text { docentes, para que ellos puedan ir más allá del } \\
\text { modelo de exposición - entrenamiento - verificación } \\
\text { y clasificación }\end{array}$ \\
\hline $\begin{array}{l}\text { (Perazzi y Celman, } \\
\text { 2017) }\end{array}$ & $\begin{array}{l}\text { Estudiar las prácticas de evaluación } \\
\text { formativa que existen en una } \\
\text { institución de formación de } \\
\text { ingenieros, para visibilizarlas. }\end{array}$ & $\begin{array}{l}\text { Se seleccionaron y estudiaron } \\
\text { cinco casos de prácticas de } \\
\text { evaluación formativa. }\end{array}$ & $\begin{array}{l}\text { Entrevistas a estudiantes docentes } \\
\text { y un referente institucional, más } \\
\text { análisis de documentos diversos. }\end{array}$ & $\begin{array}{l}\text { La evaluación formativa es aquella que es valorada } \\
\text { por los estudiantes como instancia de aprendizaje. } \\
\text { Todos los casos estudiados han sido mencionados } \\
\text { por los estudiantes entrevistados, con mayor o } \\
\text { menor frecuencia, como prácticas de evaluación } \\
\text { interesantes o significativas. }\end{array}$ \\
\hline
\end{tabular}

